Document downloaded from:

http://hdl.handle.net/10251/140910

This paper must be cited as:

Asensi Dasí, EJ.; Alemany Martínez, E.; Seco Torrecillas, A.; Ferrer, J. (31-0).

Characterization of activated sludge settling properties with a sludge collapse-acceleration stage. Separation and Purification Technology. 209:32-41.

https://doi.org/10.1016/j.seppur.2018.07.006

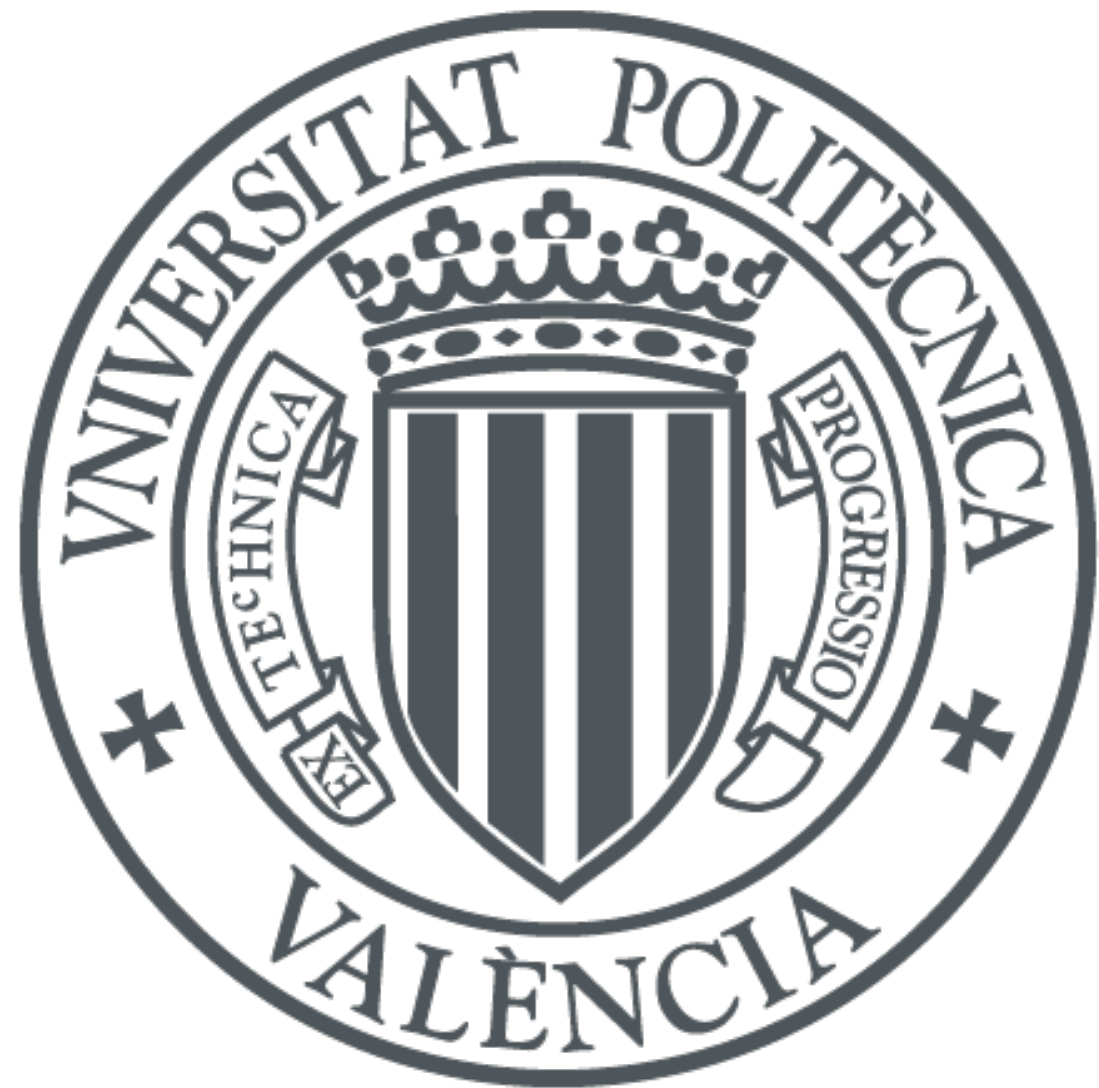

The final publication is available at

https://doi.org/10.1016/j.seppur.2018.07.006

Copyright Elsevier

Additional Information 


\title{
Characterization of activated sludge settling properties with a sludge
}

\section{collapse-acceleration stage}

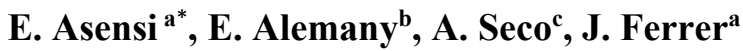

${ }^{a}$ CALAGUA, Unidad Mixta UV-UPV, Institut Universitari d'Investigació d'Enginyeria de l'Aigua i

Medi Ambient - IIAMA, Universitat Politècnica de València, Camí de Vera s/n, 46022, València, Spain (e-mail: easensi@hma.upv.es, jferrer@hma.upv.es)

${ }^{\text {b}}$ Departamento de Matemática Aplicada. Universitat Politècnica de València. Camí de Vera s/n, 46022.

València. Spain (e-mail: ealemany@mat.upv.es)

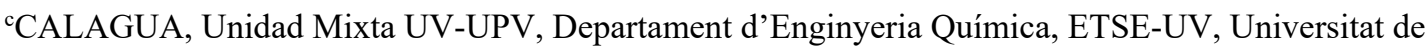
València, Avinguda de la Universitat s/n, 46100, Burjassot, València, Spain (e-mail: Aurora.Seco@uv.es)

${ }^{*}$ Corresponding author.

E-mail address: easensi@hma.upv.es (E. Asensi) 


\begin{abstract}
The sedimentability of the activated sludge can be affected by the presence of a large variety of coagulants and polymers from a previous physical-chemical process. In this paper, the activated sludge settling process in industrial wastewater treatment plants where the sludge does not settle in a conventional way is studied. The two observed constant hindered settling velocity stages and the instant the intermediate sludge acceleration period occurs are described. A variation of the Richardson and Zaki model is used to characterize the two stages of constant settling velocity. The concentration of suspended solids, where a sudden decrease of hindered settling velocity was observed, is calculated. Finally, a new hypothesis to explain the processes triggering the collapse of the initial homogeneous sludge structure and the existence of an acceleration period is formulated.
\end{abstract}

\title{
KEYWORDS
}

Hindered settling, activated sludge, Richardson-Zaki model, sludge acceleration. 


\section{INTRODUCTION}

Many secondary settling tanks design methods are based on the experience acquired in the design of urban wastewater treatment plants. It is also common to use models to simulate settling tanks by calculating the hindered settling velocity from standard values or using empirical formulas obtained from urban wastewater treatment plants. However, in some urban wastewater treatment plants, and especially in industrial ones, the sedimentability of activated sludge can be affected by the presence of coagulants and polymers used in a previous physicalchemical process.

In a batch settling test with activated sludge it is usual to find an initial induction stage of a few minutes. The increase of the settling velocity during the induction stage is due to the dissipation of the turbulence generated in the filling of the settling column [1]. The presence of cationic polymers in the sludge reduces the length of this induction period and increases the hindered settling velocity of the sludge $[2,3]$.

The existence of an induction period in settling tests with flocculate suspensions in the compression range has also been described. This induction period is due to the initial increase in the permeability of the suspension resulting from the formation of channels through which the water rises [4].

Chen et al. [5] studied the activated sludge settling velocity from an industrial wastewater treatment plant in Taiwan. In the settling tests they observed, for certain solid concentrations, two stages with constant hindered settling velocity and an intermediate stage where the acceleration of sludge occurs. They also observed the appearance of large flocs during the speed-up period starting from a homogeneous sludge.

Zhao $[6,7]$ also described the same acceleration process with primary sludge coagulated with aluminium sulphate and with an anionic organic polymer. Zhao [6] observed that the sludge acceleration was higher in the underdose range of the polymer $(<10 \mathrm{mg} / \mathrm{l})$ and that the increase of settling velocity could be due to the sludge flocculation during sedimentation. According to 
Zhao [6] in the underdose range of the polymer the sludge sedimentability was controlled by the formation of large flocs and by the progressive decrease of the viscosity. While in the overdose range of the polymer $(>10 \mathrm{mg} / \mathrm{l})$ the sludge sedimentability was controlled by the formation of a networked sludge structure surrounded by an excess of polymer. Zhao [7] considered that this networked structure links flocs and seems to cause a packing regime.

An increase in the hindered settling velocity in colloidal suspensions with weak gel structure was also reported. In a weak gel, the excess of unabsorbed polymer generates weak bonds between the colloidal particles due to the attractive forces induced by the depletion mechanism. Weak gels present an initial period of no sedimentation, or sedimentation at constant velocity, and a later period of gel collapse with a sudden increase of settling velocity [8]. The gel net undergoes restructuring and weakens over time due to the breakage and the readjustment of the bonds [9]. In these suspensions the delay time in which the collapse of the suspension occurs increases exponentially with the concentration of the unabsorbed polymer due to the increase in bond strength by the depletion mechanism [10]. Kilfoil et al. [10] also showed that an increase in the volumetric fraction of the solids causes an increase in the delay time due to the reduction of the free volume in which the unabsorbed polymer is found.

This form of sedimentation has not been studied in detail in activated sludge. Chen et al. [5] and Zhao [6] did not study the instant in which the process of acceleration of sludge takes place, nor the possible relationship between the different settling stages. However, in this study the two stages with constant hindered settling velocity, as well as the relationship between these two stages and the instant at which sludge acceleration occurs, are described and characterized. The sudden decrease in final hindered settling velocity in a small range of suspended solids concentrations, not previously described in the literature, is also studied. Finally, a mechanism is proposed to describe the collapse of the initial homogeneous sludge structure and the appearance of the stage of sludge acceleration. 


\section{MATERIALS AND METHODS}

Activated sludge samples for the tests were obtained from the industrial wastewater treatment plant Ford Spain located in Almussafes, Valencia (Spain). The plant comprises a set of physical-chemical treatments, as well as a biological treatment with an oxidation ditch. In the physical-chemical treatments a large variety of chemical reagents are used: $\mathrm{Ca}(\mathrm{OH})_{2}$, iron and aluminium salts, and both, anionic and cationic polymers.

Sludge was collected from the oxidation ditch after the surface aerators were activated to obtain a homogeneous sludge sample. The supernatant used to make the sludge dilution was collected from the secondary clarifier effluent.

\subsection{Settling Tests}

The hindered settling tests were performed using two cylindrical columns, $1.1 \mathrm{~m}$ in height and $0.12 \mathrm{~m}$ in diameter. Preliminary studies (Fig. S1 in the supplementary material) showed the need of an initial rapid stirring of the sludge for ten minutes to reproduce the settling curves and eliminate the memory effect of the sludge described by Chen et al. [5]. The initial rapid stirring of the sludge allows reproducing the hydrodynamic conditions of the treatment plant [11] where sludge is subject to high turbulences in mechanical aerators of the biological reactor before reaching the secondary clarifier.

The height of the interface in the settling test was determined as a function of time at the end of the stirring phase. A slow stirring of the sludge was not performed during the settling test to avoid interfering with the possible flocs aggregation process [5], or with the collapse of the initial sludge structure causing the acceleration stage. The sludge concentration $(X)$ was determined from the concentration of total suspended solids using the Standard Method 2540 D [12]

To consider the effect of the chemical reagents used in the wastewater treatment plant, two sets of settling tests were performed with different composition of the supernatant. In experiment A, 
the dilution of the sludge was performed using the supernatant of the secondary clarifier and in experiment B with the supernatant diluted to $75 \%$. Settling tests were also carried out by adding during the fast agitation phase, coagulants and polymers used in the physical-chemical process.

\subsection{Density and size of flocs}

Sludge samples were taken during the initial stirring process from the settling column. The flocs in this stage are called primary flocs to distinguish them from those generated during the settling process. The density and size of the primary flocs was also determined.

The density of the dry sludge $\left(\rho_{s}\right)$ was determined using a pyknometer method [13] and the primary floc density $\left(\rho_{p}\right)$ with a method based on centrifugation in homogenous density solutions [14].

Image analysis techniques were used to determine the distribution of floc size. Images were acquired by light field microscopy using an optical microscope and a digital coupled camera with a resolution of 10 megapixels. A minimum of 120 images were taken in each test to have a sample of floc images large enough to be able to perform a statistical analysis later. The analysis and processing of images was performed with a program developed with MATLAB $\left(\right.$ MathWorks $\left.{ }^{\circledR}\right)$ following the methodology used by [15]. The equivalent diameter $\left(d_{p}\right)$, that is, the diameter of a circle having the floc's projected area, was determined for each identified primary floc.

\subsection{Nonlinear regressions}

The nonlinear regressions of the models under study were performed with the program Mathematica (Wolfram ${ }^{\circledR}$ ). In all the regressions, the residuals were found to have a normal distribution using the Kolmogoroff-Smirnof test and to be randomly distributed using the run test. To compare the models, the goodness of fit was established using the Sum of Squares Residuals (SSR) and the coefficient of determination from nonlinear regressions $\left(\mathrm{R}^{2}\right)$. The 
parameters of the models were found to be significant ( $\mathrm{p}$-value $<0.05)$ and errors were estimated using the standard deviation.

\section{RESULTS AND DISCUSSION}

\subsection{Characterization of activated sludge}

A dry sludge density $\left(\rho_{s}\right)$ of $1.71 \pm 0.04 \mathrm{~g} / \mathrm{ml}$ and a primary floc density $\left(\rho_{p}\right)$ of $1.046 \pm 0.004$ $\mathrm{g} / \mathrm{ml}$ were obtained. The dry sludge density obtained is higher than typical values for urban wastewater treatment plants, i.e., $1.45 \mathrm{~g} / \mathrm{ml}$ [1]. This is due to the adsorption by the flocs of the precipitates, inert colloidal particles, coagulant residues and polymers that escape the physicalchemical process. De Clercq et al. [16] obtained similar dry sludge densities (between 1.7 and $1.9 \mathrm{~g} / \mathrm{ml}$ ) in two urban treatment plants with chemical precipitation of phosphorus.

Experimental data for the equivalent diameter of the flocs after removal of the debris was found to fit a lognormal distribution. The median of such distribution was used as a measure of the primary floc size $\left(d_{p}\right)$, since the lognormal distribution is not symmetric. A $d_{p}$ value of $9.7 \mu \mathrm{m}$ was obtained.

It was also determined that $84.8 \%$ of the flocs had a small size with an equivalent diameter smaller than $25 \mu \mathrm{m}$. This result agrees with microscope observations taken in the treatment plant, as well as with those taken in the rapid stirring phase of the settling test, where a large number of flocs with a "pin-point floc" structure was observed. This type of flocs is typical in extended aeration biological processes where flocs are characterized by being weak and easily breakable with a low turbulence.

\subsection{Setting tests results}

Fig. 1 shows the height of the interface between the sludge and the supernatant $(h)$ and the hindered settling velocity $\left(V_{S}\right)$ calculated numerically in one of the settling tests performed. An initial stage (1) with constant settling velocity $\left(V_{i}\right)$, followed by a stage of sludge acceleration 
(2) and a second and final stage (3) of constant settling velocity $\left(V_{f}\right)$ were observed. In all settling tests performed, the three stages described were observed (Fig. 2), as well as the typical hindered settling interface between the sludge and supernatant. The sludge settling stages described above appeared also when the initial rapid stirring of the sludge was not performed.

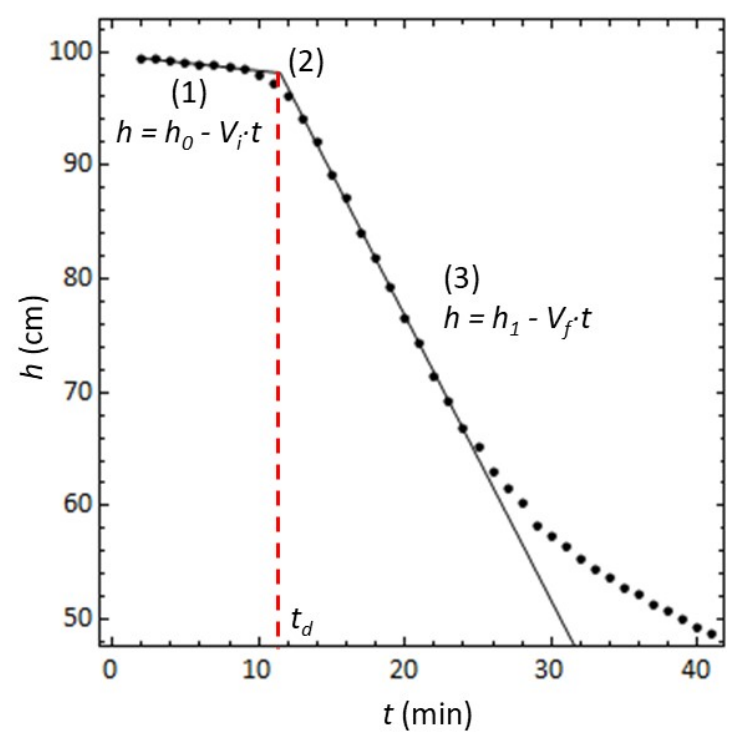

(a)

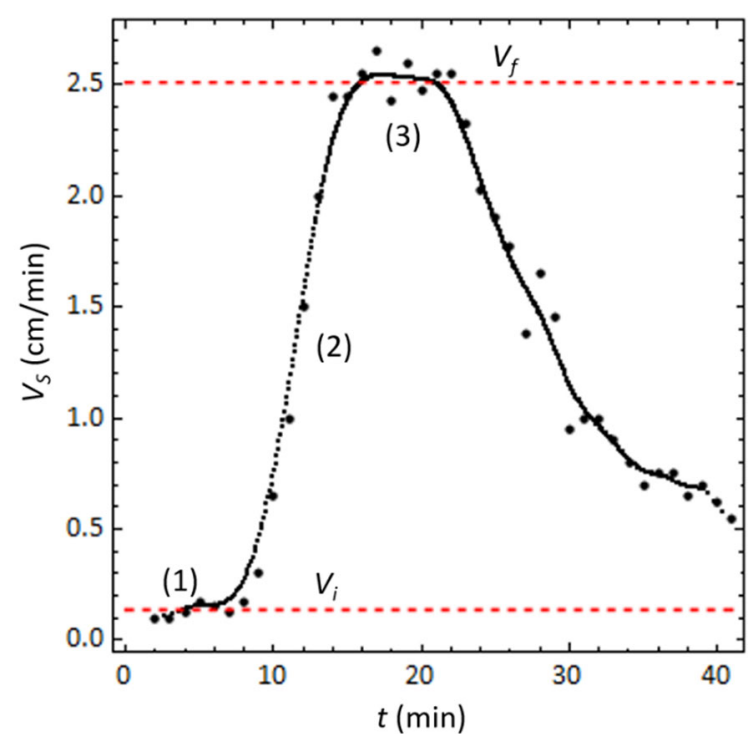

(b)

Fig. 1. Typical Settling curve $h(\mathrm{t})$ and settling velocity $V_{S}(\mathrm{t})$ of settling tests. 


\section{Experiment A}

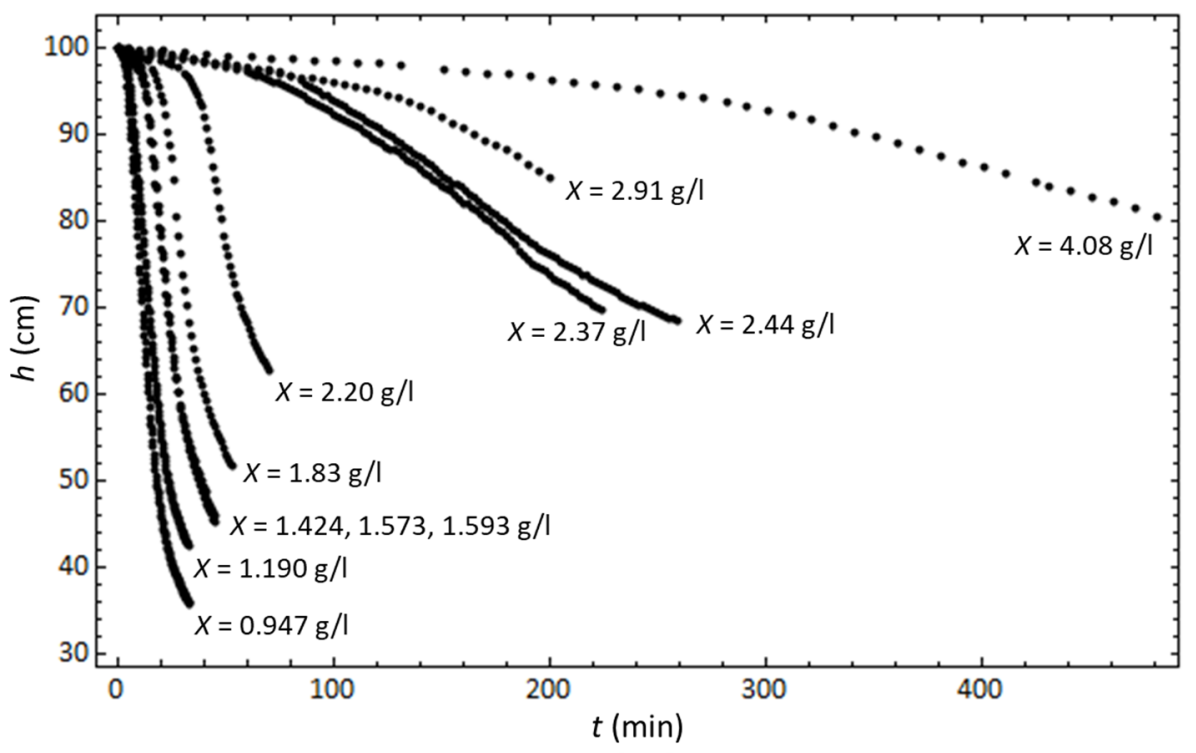

Experiment B

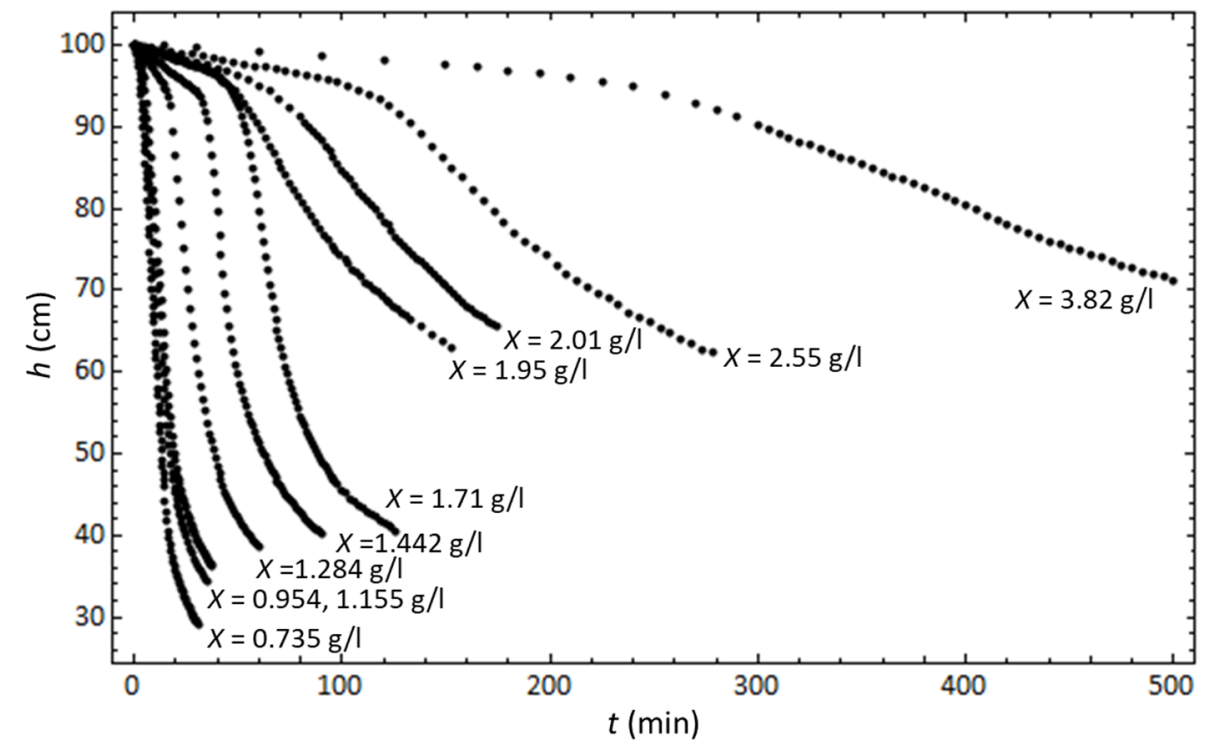

Fig. 2. Settling curves $h(\mathrm{t})$ of the tests performed in experiments A and B.

Fig. 3 shows the initial and final hindered settling velocity as a function of $X$. An abrupt decline of $V_{f}$ in a small interval of $X$ is observed. This jump is not found in the initial hindered settling velocity. This apparent discontinuity is a characteristic of the sludge under study since it appears in experiments $\mathrm{A}$ and $\mathrm{B}$, and in any other study performed with the sludge from the treatment plant. 
Experiment A
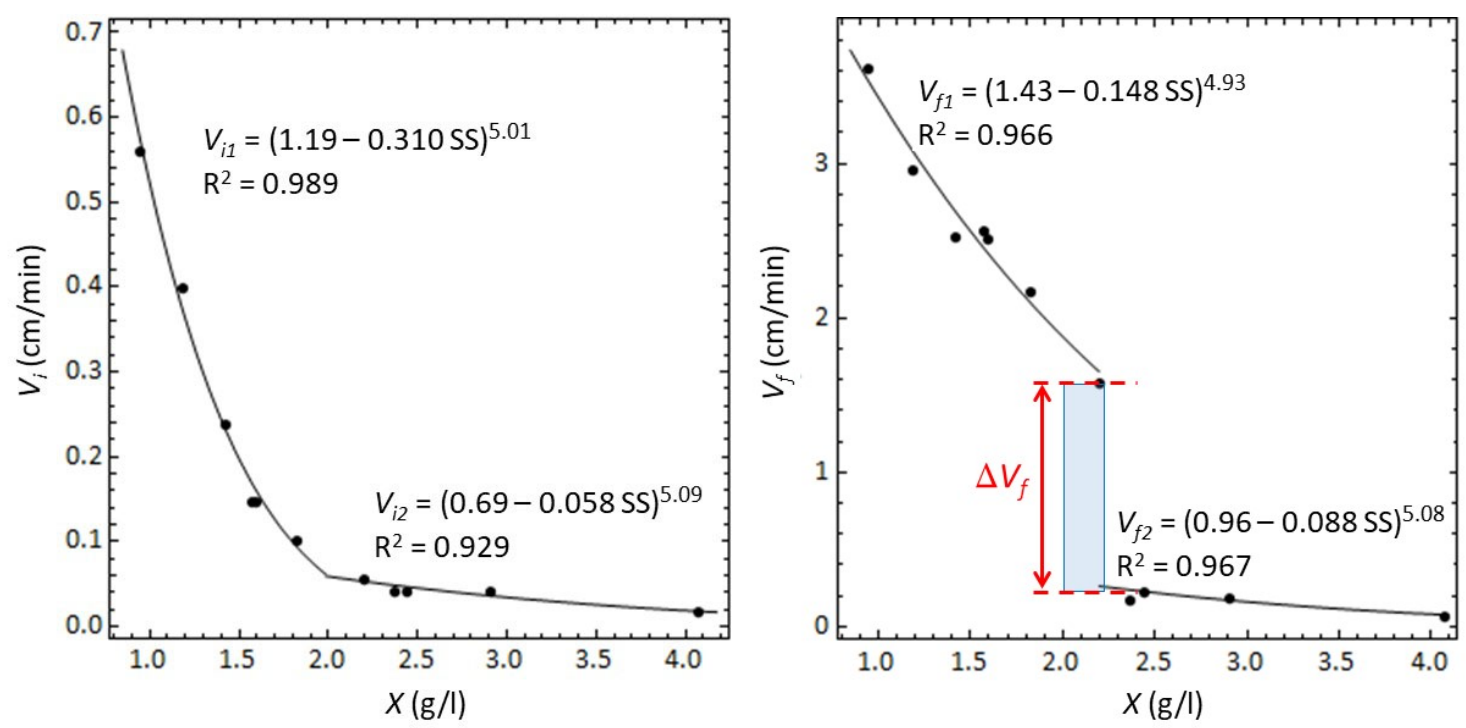

Experiment B
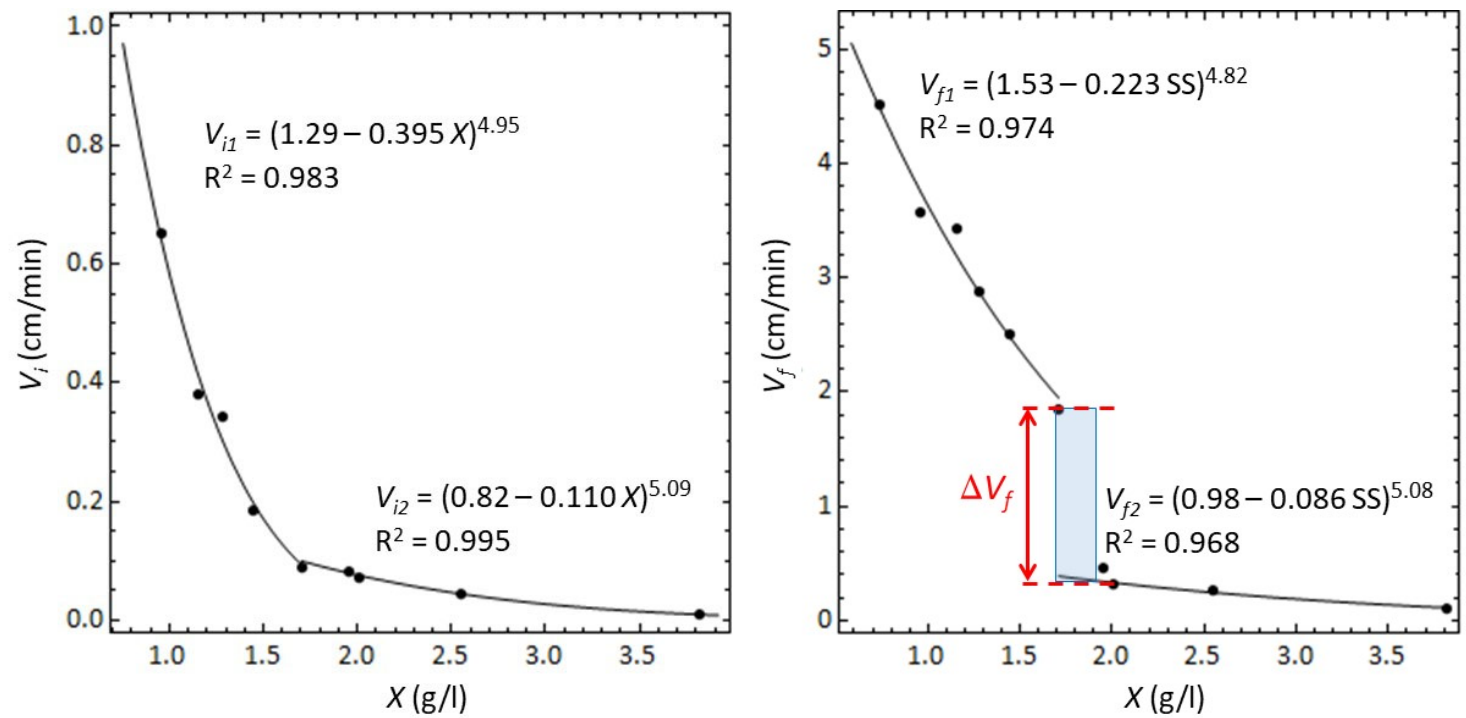

Fig. 3. Initial and final settling velocity as a function of $X$.

The delay time $\left(t_{d}\right)$ is obtained as the time corresponding to the intersection point of the two lines representing the initial and final constant settling velocity (Fig. 1a). The delay time allows estimating when the sludge accelerates and when the transition between the two stages takes place. In the tests performed, the delay time varied between three minutes and four hours and increased exponentially as $X$ increased (Fig. 4a). 


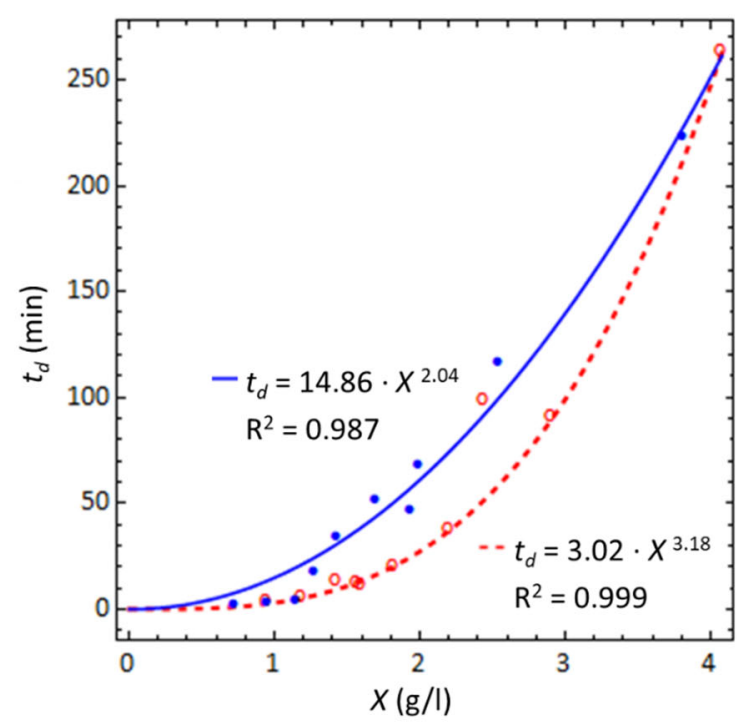

(a)

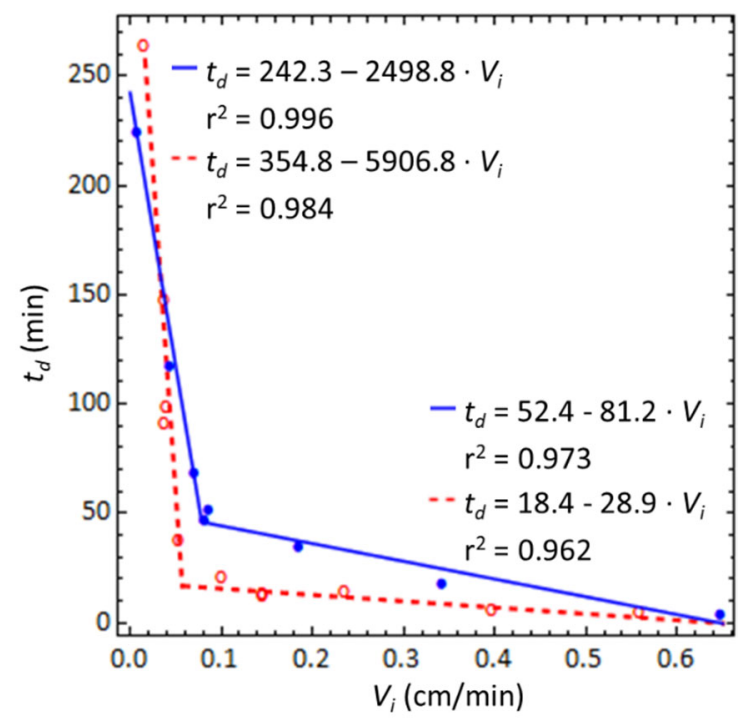

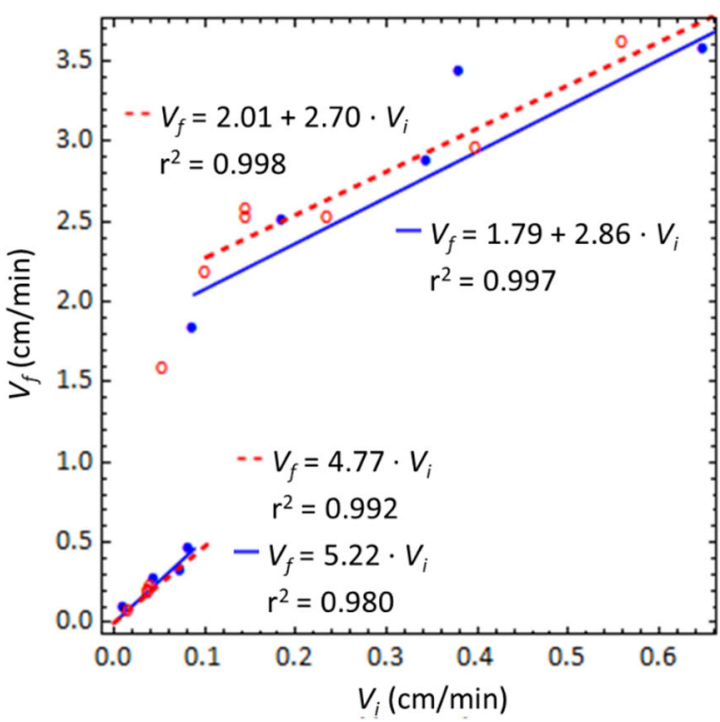

(b)

\section{- Experiment A}

- Experiment B

(c)

Fig. 4. (a) Delay time $\left(t_{d}\right)$ as a function of $X$. (b) Relationship between final settling velocity $\left(V_{f}\right)$ and initial settling velocity $\left(V_{i}\right)$. (c) Relationship between delay time $\left(t_{d}\right)$ and initial settling velocity $\left(V_{i}\right)$.

Representing $V_{f}$ versus $V_{i}$, two linear zones separated by a jump can be clearly distinguished (Fig. 4b). It is worth noting that the relative increase in settling velocity after the accelerating process is smaller for high $X$ concentrations. On the other hand, the delay time $t_{d}$ is linearly related to $V_{i}$ so that the accelerating process appears later, when the initial sludge settling velocity is lower (Fig. 4c). 
The presence of chemical compounds used in the physical-chemical treatment affects the sludge sedimentability. As an evidence, the settling velocities $\left(V_{i}\right.$ and $\left.V_{f}\right)$ and the delay time $\left(t_{d}\right)$ were found to be affected by the dilution of the supernatant used in experiment B (Figures 3 and 4). In settling tests performed maintaining the concentration of $X$ it was also verified that addition of the coagulants and polymers used in the treatment plant during the initial agitation stage affects $V_{i}, V_{f}$ and $t_{d}$ (Fig. 5).

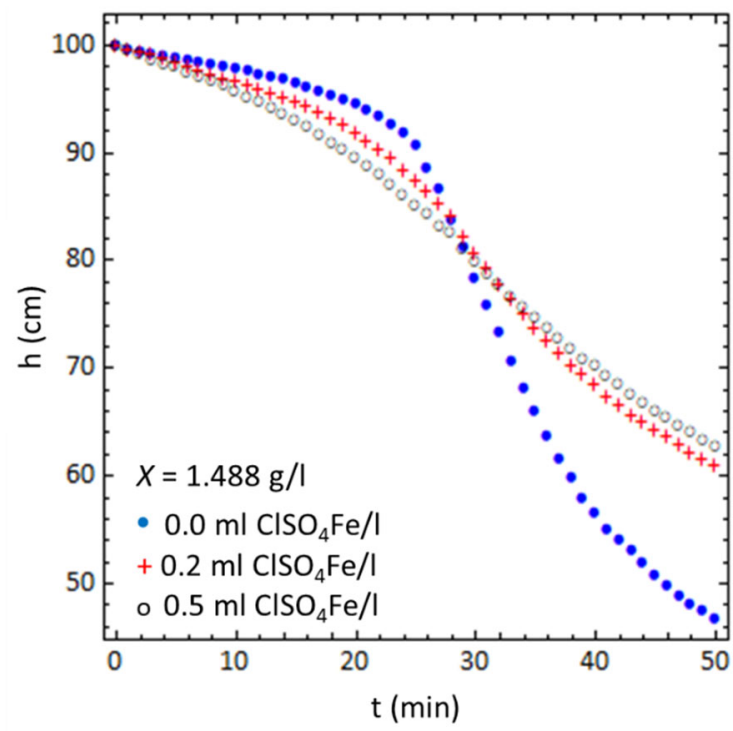

(a)

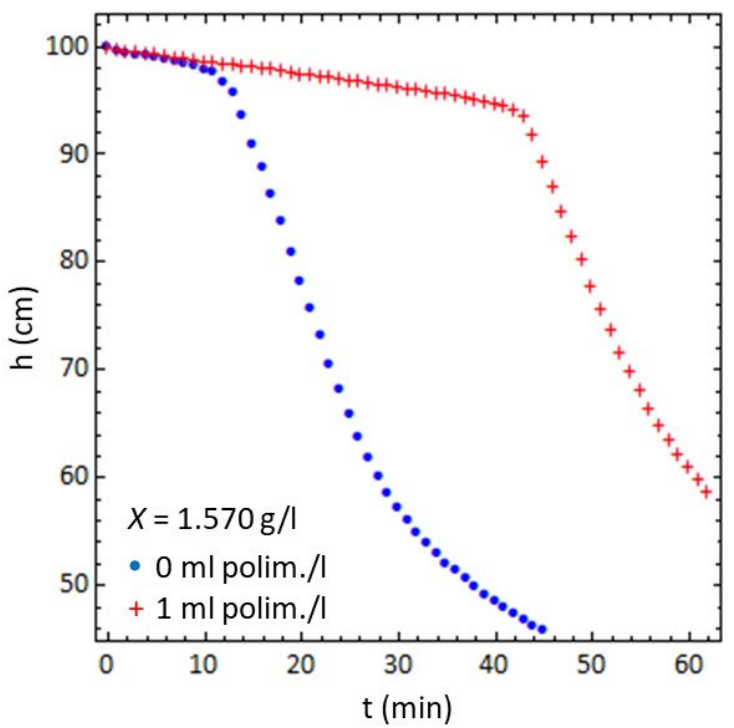

(b)

Fig. 5. Effect of coagulants and polymers used in the physical-chemical treatment on sludge sedimentability. (a) Addition of $\mathrm{ClSO}_{4} \mathrm{Fe}$. (b) Addition of the polymer Gardo TP 55.

\subsection{Modelling the initial and final settling velocity}

Some of the models most commonly used to describe the activated sludge hindered settling velocity are Vesilind's exponential model $\left(V_{S}=k \exp (-n X)\right)$, Yoshioka's potential model $\left(V_{S}=k^{-n}\right)$ and Cho's models $\left(V_{S}=k \exp (-n X) / X\right.$ and $\left.V_{S}=k(1-n X)^{4} / X\right)$ [17]. The hindered settling velocities $V_{i}(X)$ and $V_{f}(X)$ were studied considering these models and a modification of Richardson and Zaki model [18] proposed by Font et al. [19]. The latter takes into account the external porosity of the flocs and a non-laminar regime (Eq. (1)). 


$$
\begin{aligned}
& V_{s}=V_{0}\left(1-\frac{j}{\rho_{s}} X\right)^{n} \\
& \left\{\begin{array}{l}
\frac{5.09-n}{n-2.73}=0.104 R e^{0.877} \\
V_{0}=\frac{g\left(\rho_{f}-\rho\right) d_{f}^{2}}{18 \mu} \cdot \frac{1}{1+0.15 R e^{0.687}} \\
\rho_{f}=\frac{\rho_{s}+(j-1) \rho}{j}
\end{array}\right.
\end{aligned}
$$

In the modified Richardson and Zaki model, the terminal settling velocity of the flocs $\left(V_{0}\right)$ is related to the diameter $\left(d_{f}\right)$, the density of the flocs $\left(\rho_{f}\right)$, and Reynolds number $(\operatorname{Re}<500)$. The diameter of the floc is defined as the equivalent diameter of a sphere with the same volume of the floc. The density of the flocs is calculated by mass balance with Eq. (2), and Reynolds number is calculated as $\rho \cdot d_{f} \cdot V_{0} / \mu$. The aggregate volume index $(j)$ is defined as the quotient between the volume of the flocs $\left(v_{f}\right)$ and the volume of the solids contained within the flocs $\left(v_{s}\right)$. The model's exponent $(n)$ is obtained as a function of Re by Eq. (1b), proposed by Garside and Al-Dibouni [20], which is valid for volumetric fractions of flocs $\left(\phi_{f}=j \cdot X / \rho_{s}\right)$ greater than 1 .

Richardson and Zaki parameters of the model $\left(V_{0}\right.$ and $\left.j\right)$ were obtained from experimental data using nonlinear regressions of Eq. (1a) by performing an iterative process to obtain the convergence for the value of $n$. In each iteration, after obtaining $V_{0}$ and $j, d_{f}$ is calculated numerically from Eq. (3), then $R e=\rho \cdot d_{f} \cdot V_{0} / \mu$ is obtained and the parameter $n$ is recalculated from Eq. (1b). Parameters from the other models were obtained by performing nonlinear regressions. All the models considered have two independent parameters.

$$
V_{0}+0.15\left(\frac{\rho}{\mu}\right)^{0.687} V_{0}^{1.687} d_{f}^{0.687}-\frac{g\left(\rho_{S}-\rho\right)}{18 \mu} \frac{d_{f}^{2}}{j}=0
$$

The regressions of $V_{f}$ as a function of $X$ were performed considering two subintervals of $X$ due to the apparent discontinuity observed in the experimental data (Fig. 3). For the regressions of $V_{i}$ 
two subintervals of $X$ were considered too, since the regressions performed with a single range of solids showed the residuals were not random at high $X$ concentrations (Fig. S2 in the supplementary material).

From the statistical point of view, the best model to describe $V_{i}(X)$ and $V_{f}(X)$ was found to be that of Richardson and Zaki, since this model gave the lowest value of SSR (Fig. S3 in the supplementary material) as well as a normal and random distribution of the residuals. In addition, the model has the advantage of describing the hindered settling velocity as a function of the characteristics of the flocs. It should be noted that, for all the experimental fittings performed, the exponent of Richardson and Zaki model was found to have a value very close to 5 (Fig. 3). It was verified that simplifying the model and setting $n=5.0$ the regression results are the same.

\subsection{Application of Richardson and Zaki model}

Plotting $V_{i}{ }^{1 / n}$ and $V_{f}^{1 / n}$ as functions of $X$ (Fig. 6) shows that the sharp decrease of $V_{f}^{1 / n}$ agrees approximately with the slope change of $V_{i}{ }^{1 / n} . X_{t}$ is the point in the concentration of $X$ where the transition between zones with different slopes in $V_{i}$ and the sharp decrease in $V_{f}$ occurs. $X_{t}$ is calculated experimentally as the value of $X$ at which the lines corresponding to the initial settling velocities $V_{i 1}$ and $V_{i 2}$ intersect (Eq. (4c)). Thus, the model is defined considering two intervals of $X: X<X_{t}$ and $X \geq X_{t}$ (Eq. (4)).

$$
\begin{aligned}
& V_{i}(X)= \begin{cases}V_{i 1}(X)=V_{0 i 1}\left(1-\frac{j_{i 1}}{\rho_{s}} X\right)^{5} & X<X_{t} \\
V_{i 2}(X)=V_{0 i 2}\left(1-\frac{j_{i 2}}{\rho_{s}} X\right)^{5} & X \geq X_{t}\end{cases} \\
& V_{f}(X)= \begin{cases}V_{f 1}(X)=V_{0 f 1}\left(1-\frac{j_{f 1}}{\rho_{s}} X\right)^{5} & X<X_{t} \\
V_{f 2}(X)=V_{0 f 2}\left(1-\frac{j_{f 2}}{\rho_{s}} X\right)^{5} & X \geq X_{t}\end{cases} \\
& X_{t}=\rho_{s} \frac{V_{0 i 1}{ }^{1 / 5}-V_{0 i 2}{ }^{1 / 5}}{j_{i 1} V_{0 i 1}{ }^{1 / 5}-j_{i 2} V_{0 i 2}{ }^{1 / 5}}
\end{aligned}
$$


The model allows classifying the studied activated sludge sedimentability in four zones or different regimes. Fig. 6 shows four straight zones (i1, i2, f1 and f2) classified according to $X$ being greater or less that $X_{t}$ (interval 1 or 2), or depending on the constant settling velocity being the initial one or the final one. In other studies, the existence of different hindered settling regimes as a function of $X$ has also been observed [5,21]. However, the sharp decrease in the hindered settling velocity over a small range of suspended solids concentrations has not been previously described in the literature.

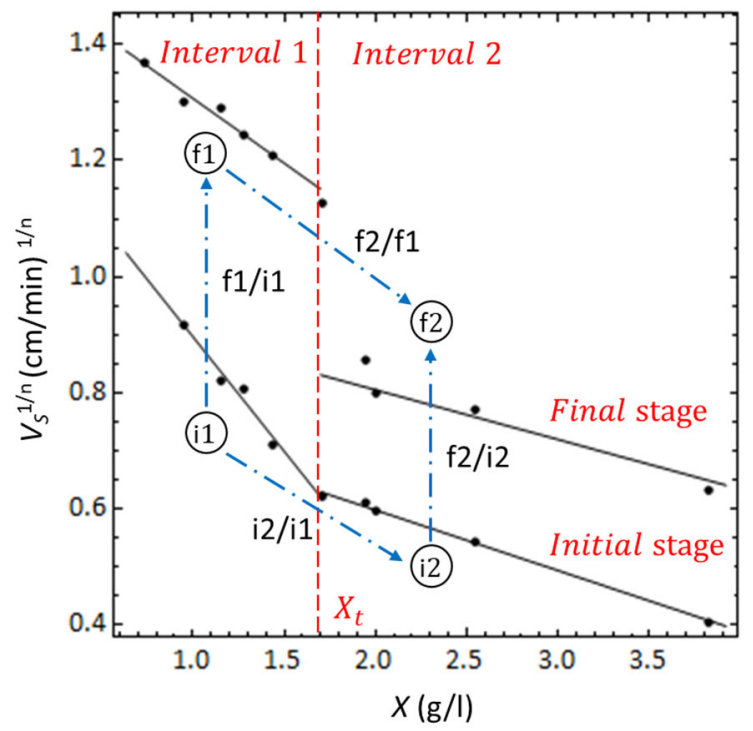

Fig. 6. Classification of sedimentation zones for the activated sludge studied.

The model used describes the activated sludge sedimentability considering an equivalent system with the same hindered settling velocity as the activated sludge, formed by spheres with a diameter equal to the mean diameter of the flocs. The model is an ideal approximation that do not take into account that activated sludge flocs have different sizes and are permeable and not spherical. Still, the model can be used to estimate the order of magnitude of the average characteristics of the flocs and to describe from them the changes in the flocs occurring after the sludge acceleration process and during the transition from $X<X_{t}$ to $X>X_{t}$. 


\subsubsection{Characteristics of flocs}

Table 1 shows the values of $d_{f}, V_{0}$, and Re calculated with the model in the different sedimentation zones. Most of the $R e$ values are outside the laminar regime $(R e<0.2)$, therefore the Richardson and Zaki model in laminar regime with $n=4.65$ [5] is not suitable for this study. Thus, it is necessary to correct $V_{0}$ and $n$ as a function of $\operatorname{Re}$ (see Eq. (1)).

\begin{tabular}{|c|c|c|c|c|}
\hline Exp. & Zone & $\begin{array}{c}d_{f} \\
(\mathbf{m m})\end{array}$ & $\begin{array}{c}V_{0} \\
(\mathrm{~mm} / \mathbf{s})\end{array}$ & $\operatorname{Re}$ \\
\hline \multirow{4}{*}{ A } & i1 & $0.70 \pm 0.04$ & $0.40 \pm 0.04$ & $0.27 \pm 0.03$ \\
\hline & $\mathrm{f} 1$ & $0.70 \pm 0.04$ & $0.98 \pm 0.06$ & $0.68 \pm 0.06$ \\
\hline & $\mathrm{i} 2$ & $0.097 \pm 0.016$ & $0.025 \pm 0.007$ & $0.0024 \pm 0.0008$ \\
\hline & $\mathrm{f} 2$ & $0.24 \pm 0.04$ & $0.14 \pm 0.04$ & $0.032 \pm 0.011$ \\
\hline \multirow{4}{*}{$\mathrm{B}$} & i1 & $0.94 \pm 0.07$ & $0.59 \pm 0.09$ & $0.55 \pm 0.10$ \\
\hline & $\mathrm{f} 1$ & $0.99 \pm 0.05$ & $1.30 \pm 0.09$ & $1.28 \pm 0.12$ \\
\hline & i2 & $0.192 \pm 0.013$ & $0.062 \pm 0.008$ & $0.012 \pm 0.002$ \\
\hline & $\mathrm{f} 2$ & $0.38 \pm 0.03$ & $0.27 \pm 0.04$ & $0.104 \pm 0.017$ \\
\hline
\end{tabular}

Table 1. Characteristics of flocs calculated from Richardson and Zaki model in the different settling zones.

The results obtained allowed to verify that the conditions necessary to apply the model in the four areas under study $\left(R e<500\right.$ and $\left.\phi_{f}>0.1\right)$ were fulfilled. The effect of the settling column walls was also found to be negligible. Using the equation proposed by Garside and Al-Dibouni [20], the relative error in $V_{0}$ due to the settling column diameter was determined to be between 0.2 and $2 \%$.

The model allows estimating some additional properties of the flocs. The porosity of flocs $\left(\varepsilon_{f}\right)$ is calculated as the fraction between water volume contained in the floc $\left(v_{f}-v_{s}\right)$ and the floc volume $\left(v_{f}\right)($ Eq. $(5))$. The floc's porosity due to the larger pores can be estimated from the porosity generated by the external water of the primary flocs composing the floc $\left(\varepsilon_{e x t}\right)$. This porosity is calculated by considering the volume of the primary flocs $\left(v_{p}\right)$ contained within the floc (Eq. (6)). Table 2 shows the values of $\varepsilon_{f}$ and $\varepsilon_{e x t p}$ calculated with Eq. (5) and (6). 


$$
\begin{aligned}
& \varepsilon_{f}=\frac{v_{f}-v_{s}}{v_{f}}=1-\frac{1}{j} \\
& \varepsilon_{\text {ext } p}=\frac{v_{f}-v_{p}}{v_{f}}=1-\frac{\rho_{s}-\rho}{j\left(\rho_{p}-\rho\right)}
\end{aligned}
$$

\begin{tabular}{cccc}
\hline \multirow{2}{*}{ Exp. } & Zone & $\boldsymbol{\varepsilon}_{f}$ & $\boldsymbol{\varepsilon}_{\text {ext }}$ \\
\hline \multirow{3}{*}{$\mathrm{A}$} & & & \\
\cline { 2 - 4 } & $\mathrm{i} 1$ & $0.9977 \pm 0.0002$ & $0.966 \pm 0.004$ \\
\cline { 2 - 4 } & $\mathrm{f} 1$ & $0.9941 \pm 0.0006$ & $0.912 \pm 0.012$ \\
\cline { 2 - 4 } & $\mathrm{i} 2$ & $0.993 \pm 0.002$ & $0.89 \pm 0.03$ \\
\cline { 2 - 4 } & $\mathrm{f} 2$ & $0.994 \pm 0.002$ & $0.91 \pm 0.03$ \\
\hline \multirow{3}{*}{$\mathrm{B}$} & $\mathrm{i} 1$ & $0.9981 \pm 0.0002$ & $0.971 \pm 0.004$ \\
\cline { 2 - 4 } & $\mathrm{f} 1$ & $0.9958 \pm 0.0004$ & $0.938 \pm 0.008$ \\
\cline { 2 - 4 } & $\mathrm{i} 2$ & $0.9957 \pm 0.0004$ & $0.935 \pm 0.009$ \\
\cline { 2 - 4 } & $\mathrm{f} 2$ & $0.9950 \pm 0.0005$ & $0.926 \pm 0.011$ \\
\hline
\end{tabular}

Table 2. Flocs porosities calculated from Richardson and Zaki model in the different settling zones.

The characteristics of the flocs obtained using Richardson and Zaki model, and shown in Table 1 and Table 2, were compatible with the experimental values reported by the literature of activated sludge flocs.

The $\varepsilon_{f}$ values in the settling zones under study are compatible with the typical values of porosity of the activated sludge flocs calculated by mass balance. Chung and Lee [22] obtained activated sludge porosities between 0.98 and 0.99 , while Chu and Lee [23] considered that the porosity can be even higher than 0.99. Xiao et al. [24] obtained that the porosity of the activated sludge flocs after a flocculation process was between 0.91 and 0.98 . These results are compatible with those obtained for floc porosity due to primary flocs $\left(\varepsilon_{\text {ext }}\right)$.

Hribersek et al. [25] obtained that flocs with an equivalent diameter ranging between 0.15 and $1.7 \mathrm{~mm}$, had terminal settling velocity of the activated sludge flocs $\left(V_{0}\right)$ between 0.2 and 2.1 $\mathrm{mm} / \mathrm{s}$. Whereas Xiao et al. [24] obtained that flocs with sizes between 1.3 and $3.7 \mathrm{~mm}$, had $V_{0}$ values between 1.9 and $7.0 \mathrm{~mm} / \mathrm{s}$. The terminal velocity of the flocs in the initial settling stage 
(zones i1 and i2) is much lower than the typical value for activated sludge with similar floc size. However, after the sludge acceleration process during the final settling stage (zones f1 and f2), $V_{0}$ has the same order of magnitude as the expected value for activated sludge.

If it is assumed that all solids are contained within the flocs, then the mean number of flocs per unit volume $\left(n_{f}\right)$ can be estimated as the ratio of the solids volumetric fraction $\left(\phi_{s}=X / \rho_{s}\right)$ to the mean volume of solids contained in a floc $\left(v_{s}\right)$. Eq. (7) allows calculating, in the four areas under study, the mean number of flocs per unit mass of $X$ as $n_{f} / X$.

$$
\frac{n_{f}}{X}=\frac{6}{\pi \rho_{s}} \frac{j}{d_{f}^{3}}
$$

Table 3 shows the calculated ratios between the different settling zones for $d_{f}, V_{0}$ and $n_{f} / X$. The calculated ratios between the final and initial stages of interval 1 (f1/i1) and interval 2 (f2/i2) allow studying the changes produced in the flocs after the sludge acceleration process. The calculated ratios in the intervals 2 and 1 of the initial velocity stage (i2/i1), and the final stage (f2/f1) allow studying the difference between the initial and final characteristics of the flocs as a function of $X$.

\begin{tabular}{ccccc} 
Exp. & Ratio & $\boldsymbol{r} \boldsymbol{d}_{\boldsymbol{f}}$ & $\boldsymbol{r} \boldsymbol{V}_{\boldsymbol{o}}$ & $\boldsymbol{r} \boldsymbol{n}_{\boldsymbol{f}} / \boldsymbol{X}$ \\
\hline \multirow{3}{*}{$\mathrm{A}$} & $\mathrm{f} 1 / \mathrm{i} 1$ & 1.0 & 2.5 & 0.4 \\
\cline { 2 - 5 } & $\mathrm{f} 2 / \mathrm{i} 2$ & 2.5 & 5.6 & 0.08 \\
\cline { 2 - 5 } & $\mathrm{i} 2 / \mathrm{i} 1$ & 0.1 & 0.06 & 116.9 \\
\cline { 2 - 5 } & $\mathrm{f} 2 / \mathrm{f} 1$ & 0.3 & 0.1 & 22.6 \\
\hline \multirow{3}{*}{$\mathrm{B}$} & $\mathrm{f} 1 / \mathrm{i} 1$ & 1.1 & 2.2 & 0.4 \\
\cline { 2 - 5 } & $\mathrm{f} 2 / \mathrm{i} 2$ & 2.0 & 4.4 & 0.1 \\
\cline { 2 - 5 } & $\mathrm{i} 2 / \mathrm{i} 1$ & 0.2 & 0.1 & 53.6 \\
\cline { 2 - 5 } & $\mathrm{f} 2 / \mathrm{f} 1$ & 0.4 & 0.2 & 14.2 \\
\hline
\end{tabular}

Table 3. Ratios of flocs characteristics from different settling zones. 


\subsubsection{Initial hindered settling stage and calculation of $X_{t}$}

Richardson and Zaki model explains the difference between the initial sludge structure in zones i1 and i2 and lets calculate the $X_{t}$ value where the sudden decrease of $V_{f}$ occurs.

The external porosity of the flocs calculated with respect to the total suspension volume $\left(\varepsilon^{\prime}\right.$ ext $\left.f\right)$ represents the porosity due to water outside the flocs (Eq. (8)). Whereas the internal porosity of the flocs due to the larger size pores calculated with respect to the total suspension volume $\left(\varepsilon^{\prime}{ }_{e x t}\right.$ ${ }_{p}$ ) is obtained considering the total volume of water external to the primary flocs contained within the flocs (Eq. (9)). External porosity of flocs decreases with increasing $X$ because the space between the flocs decreases, whereas the internal porosity of flocs increases due to the increase in the number of flocs.

$$
\begin{aligned}
& \varepsilon_{\text {ext } f}^{\prime}=1-\phi_{f}=1-j \frac{X}{\rho_{s}} \\
& \varepsilon_{\text {ext } p}^{\prime}=\varepsilon_{\text {ext } p} \phi_{f}=\left(j-\frac{\rho_{s}-\rho}{\left(\rho_{p}-\rho\right)}\right) \frac{X}{\rho_{s}}
\end{aligned}
$$

When the initial agitation process in the settling test ends, a brief period of induction occurs. In this period, the combined effect of the dissipation of the turbulence and the beginning of the settling process with large fluctuations in the settling velocity [11], leads to a flocculation process. Therefore, flocs more porous and larger than the primary ones are generated in zones i1 and i2. The differences in the initial flocs characteristics in experiments A and B can be explained by the effect on the flocculation process of the different composition of salts and polymers in the supernatant.

If, during the initial induction period, the external porosity of the growing flocs $\left(\varepsilon_{\text {ext } f}^{\prime}\right)$ is greater than the internal porosity $\left(\varepsilon_{\text {ext }}^{\prime}\right)$, the flow of water outside the flocs is larger than the internal flow. In this case, the sludge settling and formation of flocs are both determined by the external porosity of the flocs. However, if the external and internal porosity have the same order, the 
internal flow plays an important role, so that flocs formation and sludge settling is conditioned by the internal porosity of the flocs. In this situation, the flocs will rearrange differently during the induction stage, forming an initial flocs structure different from the previous one. In addition, since the distance between the formed flocs and the size of their pores are the same order, bridges and joints between flocs can arise, generating a structure similar to a weak gel.

The mechanisms of floc formation described above are compatible with those described by Zhao [7]. Thus, depending on the $X$ concentration, initially either large flocs or a weak gel structure of smaller flocs will be formed. Richardson and Zaki model describes these two situations considering that the number of flocs per mass of $X$ formed is greater in zone i2 than in zone il (between 54 and 117 times greater) and the size of flocs smaller (between 0.1 and 0.2 times smaller) (Table 3).

The $X$ concentration that separates the two situations described above corresponds to the point where the transition between zone i1 and i2 occurs. The value of $X_{t}$ can be estimated as the concentration of $X$ where $\varepsilon^{\prime}{ }_{\text {ext }}=\varepsilon^{\prime}$ ext $^{\prime}$ in zone i1 (Eq. (10)). The $X_{\mathrm{t}}$ errors, experimental and from the model, have been calculated from Eq. (4c) and (10), considering the experimental errors of $\rho_{s}$ and $\rho_{p}$, and the estimated errors of the parameters $V_{0}$ and $j$ of the model. In Table 4 , the $X_{\mathrm{t}}$ values obtained with the model are compared with the values obtained experimentally. The results show that the model allows estimating the value of $X_{\mathrm{t}}$ where the sudden decrease in $V_{f}$ occurs.

$$
X_{t}=\frac{\rho_{s}}{2 j_{i 1}-\frac{\rho_{s}-\rho}{\rho_{p}-\rho}}
$$

\begin{tabular}{ccc}
\hline Exp. & $\begin{array}{c}\boldsymbol{X}_{\boldsymbol{t}}(\mathbf{g} / \mathbf{l}) \\
\text { Experimental }\end{array}$ & $\begin{array}{c}\boldsymbol{X}_{\boldsymbol{t}}(\mathbf{g} / \mathbf{l}) \\
\text { Richardson and Zaki }\end{array}$ \\
\hline $\mathrm{A}$ & $2.0 \pm 0.3$ & $1.97 \pm 0.10$ \\
\hline $\mathrm{B}$ & $1.7 \pm 0.2$ & $1.68 \pm 0.09$ \\
\hline
\end{tabular}

Table 4. $X_{t}$ calculated experimentally and with Richardson and Zaki model. 
It is important to note that both methods used for the calculation of $X_{t}$ are independent.

Experimentally, $X_{t}$ is obtained from the results of settling tests of zones i1 and i2 (Eq. (4c)), while the model calculates $X_{t}$ from $j_{i l}$ obtained from the settling tests in zone il and from experimental measurements of $\rho_{s}$ and $\rho_{p}$ which are independent of the settling tests.

\subsubsection{Final hindered settling stage and sludge acceleration}

The initial hindered settling stage and the acceleration process cannot be considered an induction stage due to the dissipation of the initial turbulence. The induction phase usually lasts a few minutes whereas, in tests conducted at high solids concentrations, the initial settling stage lasted several hours (Figures 2 and $4 \mathrm{a}$ ). In addition, an induction phase before the initial settling velocity stage was observed in the settling tests.

It was verified that the acceleration phase is not due to the induction process described by [4] since all the tests were carried out with concentrations of $X$ outside the compression range. Sedimentation by compression takes place from a concentration of $X\left(X_{c}\right)$ where the flocs begin to be in contact with each other. The settling tests were performed with an $X$ concentration $(1<$ $X<4 \mathrm{~g} / \mathrm{l})$ lower than the typical $X_{c}$ value for activated sludge which is between 5 and $10 \mathrm{~g} / 1$ [26]. It is also possible to estimate $X_{c}=0.64 \cdot \rho_{s} / j$ assuming that when the contact between the flocs begins, $\phi_{f}$ must approach the volumetric fraction in a fixed bed of spheres $\left(\phi_{f}=0.64\right)$. Font et al. [19] verified that this approximation is correct in metal hydroxide suspensions. The approximation was used to verified that in all settling zones being studied $X<X_{c}$. On the other hand, although a weak gel structure can result from the polymer excess, the structure of the sludge will be completely different from that generated by physical contact of the flocs in the compression range. The mechanism described by Font and Pérez [4] is based on the initial formation of channels in the sludge and does not explain the beginning of the acceleration process after a long period with constant settling velocity. 
The appearance of the period of increasing velocity cannot be explained by a conventional mechanism of flocculated sedimentation. However, there are similarities between the collapse of the colloidal weak gels and the acceleration process observed in the activated sludge.

There must be a mechanism triggering the flocculation process after a period of constant settling velocity, lasting a short time interval. The sludge acceleration phase may be caused by the fragmentation of the flocs or by the fragmentation of the weak gel structure generated after the initial rapid stirring stage. This fragmentation process can be explained by the existence of physical-chemical mechanisms that cause the rupture of bonds between primary flocs.

The flocs formed during the initial induction process and during the acceleration phase can be considered fragile because the joints between the primary flocs are weak and unstable. Proof of this is that the memory effect observed in the settling tests is easily eliminated with the initial agitation of the sludge and that flocs break easily during the tests when entering the compression zone.

Considering that the bonds between primary flocs are weak, the water flow in the areas where porosity is higher can lead to the rupture of the weak bonds that exist between the primary flocs. The rupture of bonds in the more porous areas will increment the porosity of these zones, increasing the water flow and the fragmentation process. On the other hand, François et al. [11] showed that there are large fluctuations in the flocs settling velocity with a mean velocity equal to the activated sludge hindered settling velocity. These fluctuations can generate shear forces causing a slow breakage of weak bonds between flocs of a weak gel structure. The greater the initial settling velocity of the sludge the more important the physical mechanisms of fragmentation will be. These mechanisms explain the delay in the beginning of the acceleration process with increasing $X$ due to the decrease of $V_{i}$, and the linear relationship between $t_{d}$ and $V_{i}$.

The fragmentation process can also be caused by the instability of the primary flocs bonds. This hypothesis is compatible with the linear relationship between $t_{d}$ and $V_{i}$, which suggests that sludge fragmentation $\left(t_{d}>0\right)$ also occurs when $V_{i}=0$. The chemical breakage of bonds explains 
the fragmentation process in tests with high concentration of $X$ where the initial settling velocity is very low. Another aspect supporting the hypothesis of the existence of a chemical mechanism is the dependence of the parameters relating linearly $t_{d}$ with $V_{i}$ with the composition of the supernatant.

Some mechanisms described in the literature may explain the sludge fragmentation over time when $V_{i}$ is very low. Depletion mechanisms may be important if considering the presence of debris particles, small flocs and polymers non-adsorbed by flocs in the sludge coming from the physical-chemical treatment. Depletion flocculation can generate a weak gel that weakens progressively due to the breakage of the gel bonds in a similar way to the process described by Bartlett et al. [9] in colloidal suspensions. There could be also endogenous syneresis processes within the flocs or shear densification mechanisms, so that the flocs contraction originated by the expulsion of water [27] favors the rupture of bonds between primary flocs. The densification process can be caused by the shear due to the buffeting of flocs in sedimentation [28] and/or to the external and internal water flow through the flocs.

The physical and chemical mechanisms described above can cause the fragmentation of the sludge structure and trigger a flocculation process leading to the acceleration of sludge. Finally, when a new equilibrium is reached in the floc structure, the stage with final constant hindered settling velocity begins. The results on the characteristics of flocs obtained in the final settling stage can be explained by the proposed mechanisms of fragmentation and flocs formation. Consequently, the number of flocs per unit volume decreases after the acceleration process $\left(n_{f 1} \approx 0.4 \cdot n_{i 1}\right.$ and $n_{f 2} \approx 0.1 \cdot n_{i 2}$, Table 3$)$.

For low $X$ concentrations (zones i1 and $\mathrm{fl}$ ) the physical breakage of flocs will predominate since $V_{i}$ is high. Fragmentation will occur at those few points where the larger pores were initially located, generating large fragments of the flocs. In addition, with an increase of the initial settling velocity, the number of collisions and joints between flocs will increase too. The process of aggregation of flocs will generate eventually large flocs. 
For high $X$ concentrations (zones i2 and $\mathrm{f} 2$ ) the chemical mechanism of flocs breakage will predominate since $V_{i}$ is low. The flocs breakage mechanism becomes more homogeneous, so that fragmentation occurs at many points leading to small size fragments. In addition, the flocculation process decelerates, generating fewer collisions among the flocs due to the lower settling velocity. Eventually, the aggregation of flocs leads to more compact and smaller flocs. The fragmentation and flocculation processes slow down, causing the start of the sludge acceleration phase to be delayed (high $t_{d}$ ).

The sudden jump in the final hindered settling velocity (Figures 3 and $4 \mathrm{~b}$ ) is also observed in $V_{0}$ when moving from zone $\mathrm{f} 1$ to zone $\mathrm{f} 2\left(\mathrm{r} V_{0}\right.$ between 0.1 and 0.2 , Table 3$)$. The differences between the initial sludge characteristics in zones i1 and i2 and the conversion from a predominant physical mechanism for the sludge fragmentation to a chemical mechanism, explain the formation of flocs with different characteristics in zones $\mathrm{f} 1$ and $\mathrm{f} 2$ and the subsequent decrease of $V_{0}$. On the other hand, the possible existence of a weak gel structure in the initial settling stage explains that the order of magnitude of $V_{0}$ at this stage be smaller than the typical value for the activated sludge flocs.

The proposed Richardson and Zaki model does not allow calculating the final settling velocity in the transition between zones $\mathrm{f} 1$ and $\mathrm{f} 2$ considering a discontinuity in $X_{\mathrm{t}}$ (Eq. (4)). Nor can it explain the presence of several millimeters size flocs observed in zone f1. To improve the description of sludge settling in the final settling velocity stage, it is necessary to improve the model taking into account the fractal structure of flocs, their sphericity and the effect of water flow through the flocs.

\section{CONCLUSIONS}

Two different stages with constant hindered settling velocity and an intermediate acceleration process are observed during the settling tests. 
The defined delay time allows estimating the instant when the sludge collapse-acceleration process occurs. The delay time increases exponentially with increasing $X$ concentration and decreases linearly with increasing initial hindered settling velocity.

Richardson and Zaki model lets characterize and classify the activated sludge sedimentability in a settling test with a sludge acceleration stage. It also allows estimating the $X$ concentration where a sudden decrease of final hindered settling velocity, not previously described in the literature, occurs.

The existence of two constant hindered settling velocity stages and a sludge acceleration stage can be explained by the fracture of the initial homogeneous sludge structure and the flocculation of the generated flocs.

The proposed physical-chemical mechanisms of fragmentation and the initial sludge structure explain the sudden drop observed in the final settling velocity and the delay in the start of the sludge acceleration phase.

The additives added in the previous physical-chemical treatment play an important role in the sedimentation process and in the fragmentation of the initial homogeneous sludge structure.

\section{ACKNOWLEDGEMENTS}

This research did not receive any specific grant from funding agencies in the public, commercial, or not-for-profit sectors. 


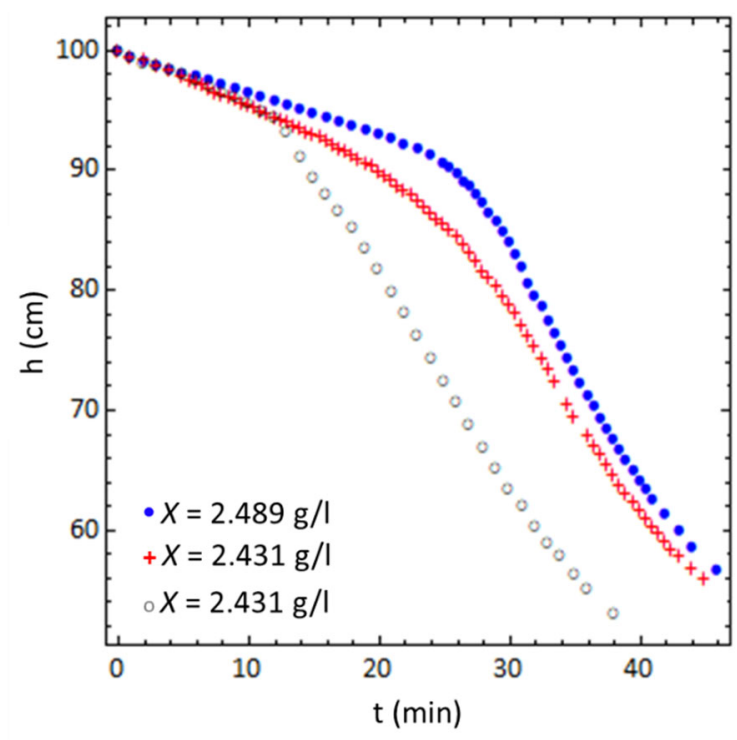

(a)

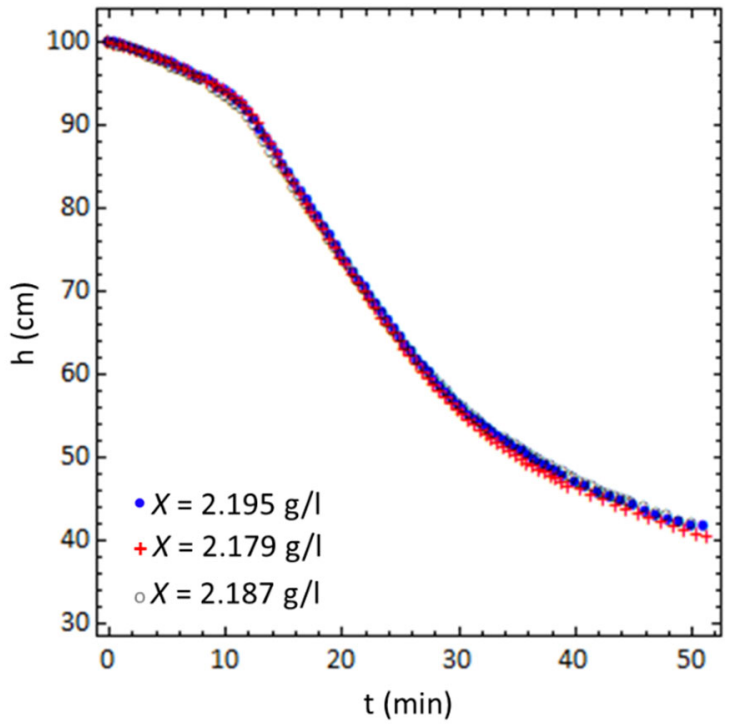

(b)

Fig. S1. Results from settling tests. (a) Without previous stirring. (b) With $10 \mathrm{~min}$ of previous stirring. 


\section{Experiment A}
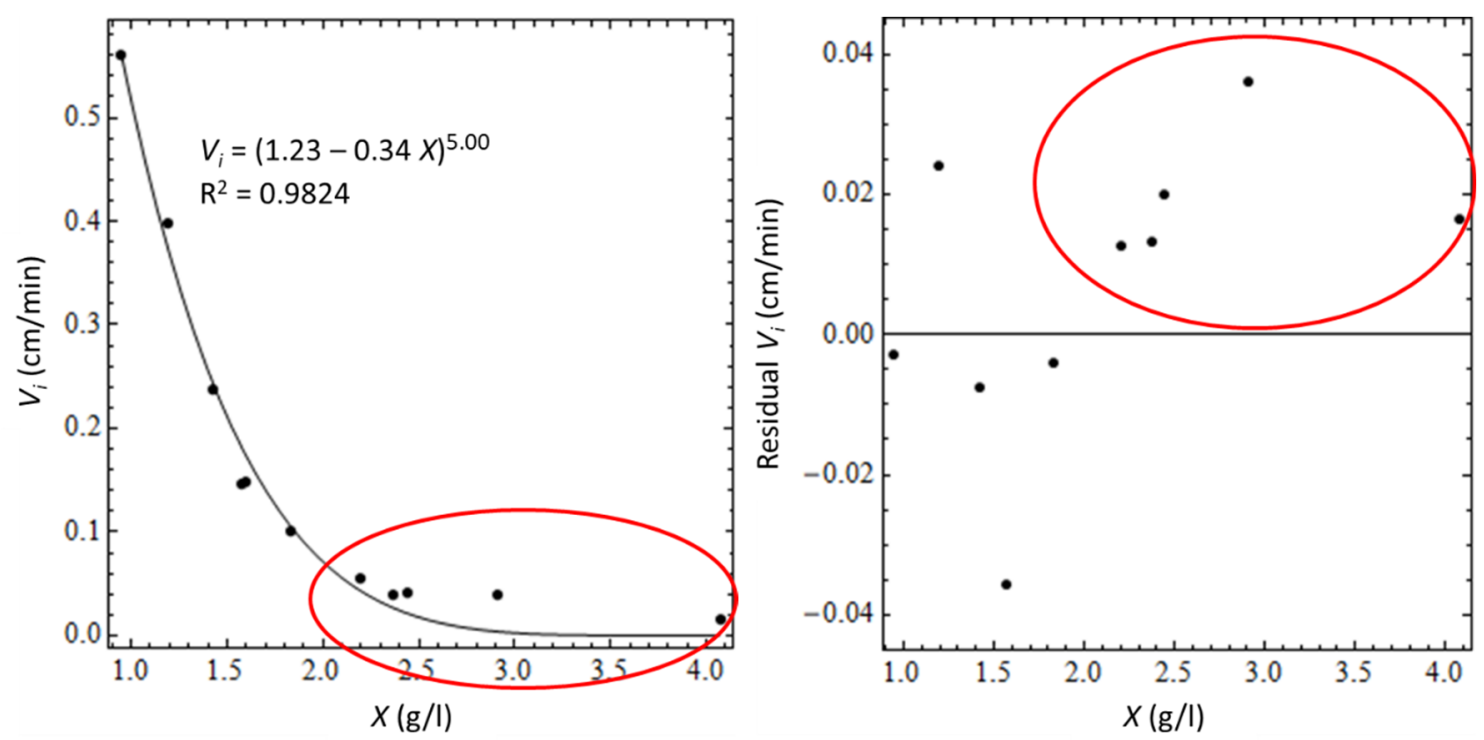

Experiment B
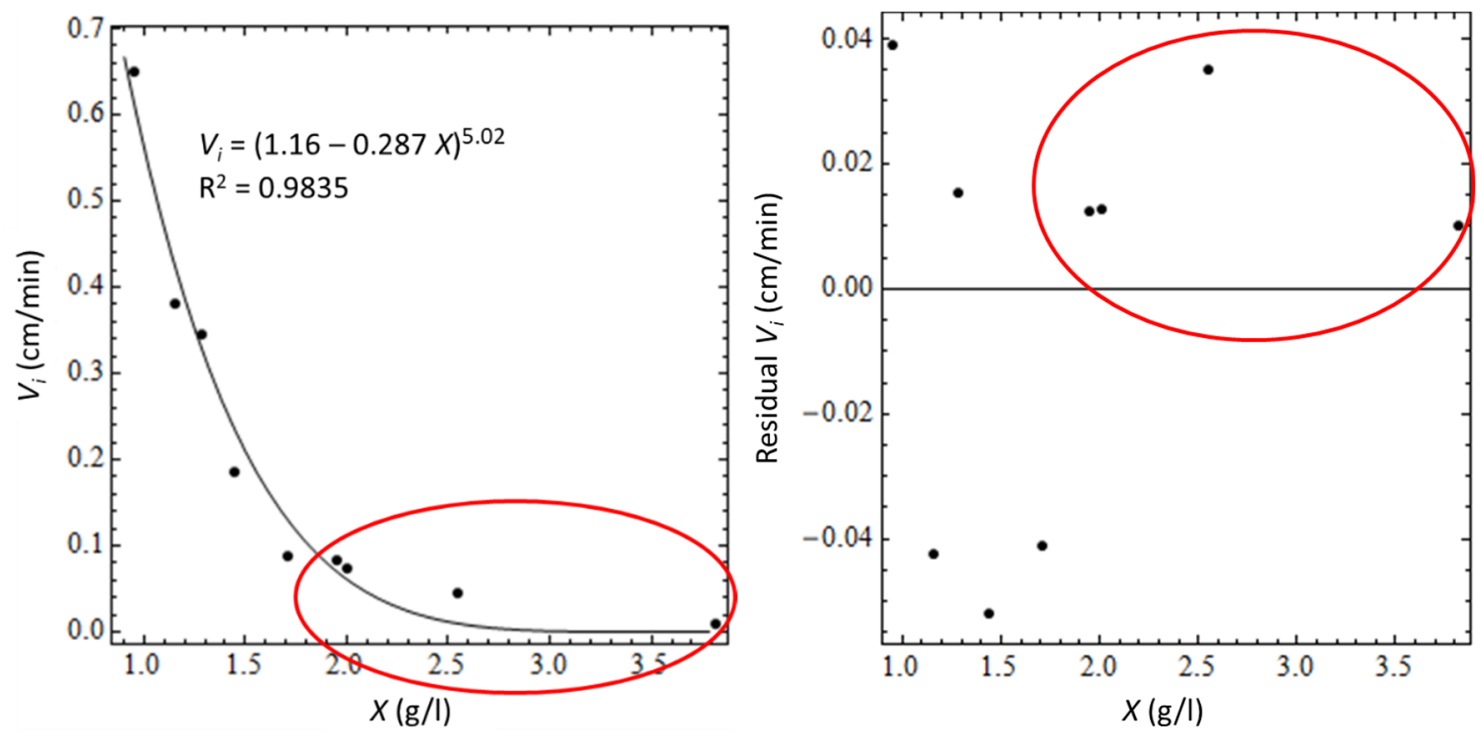

Fig. S2. Results from initial settling velocity regressions and residuals from Richardson and Zaki model. 


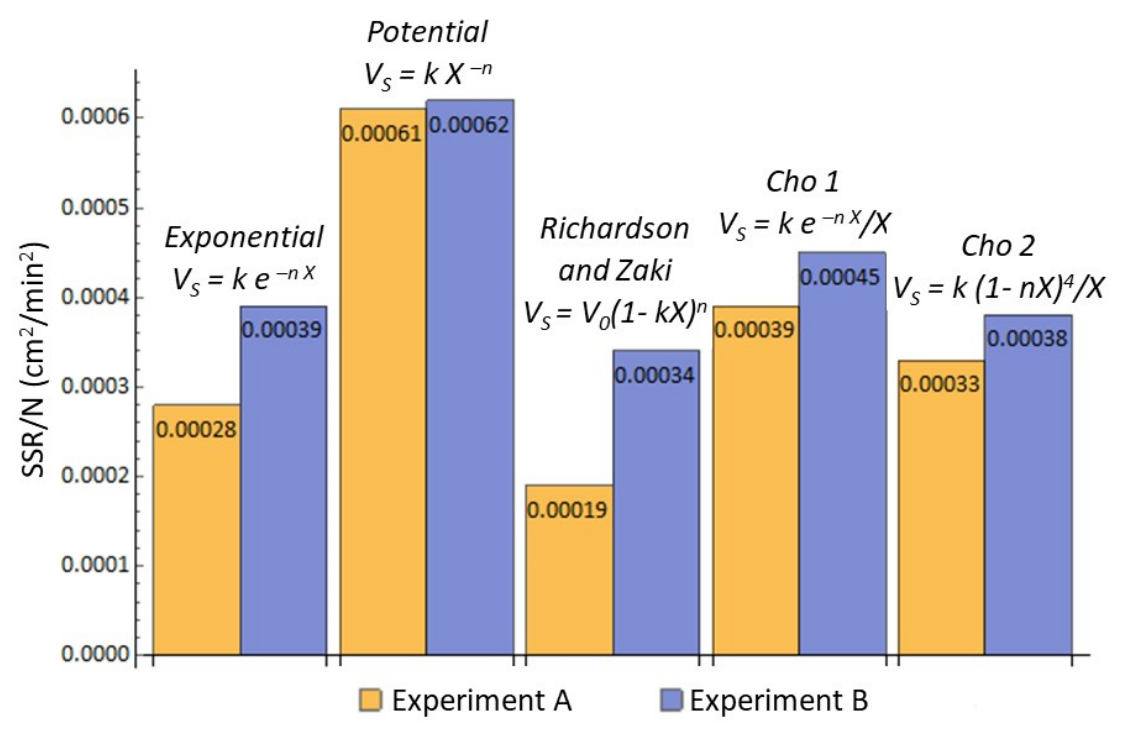

(a)

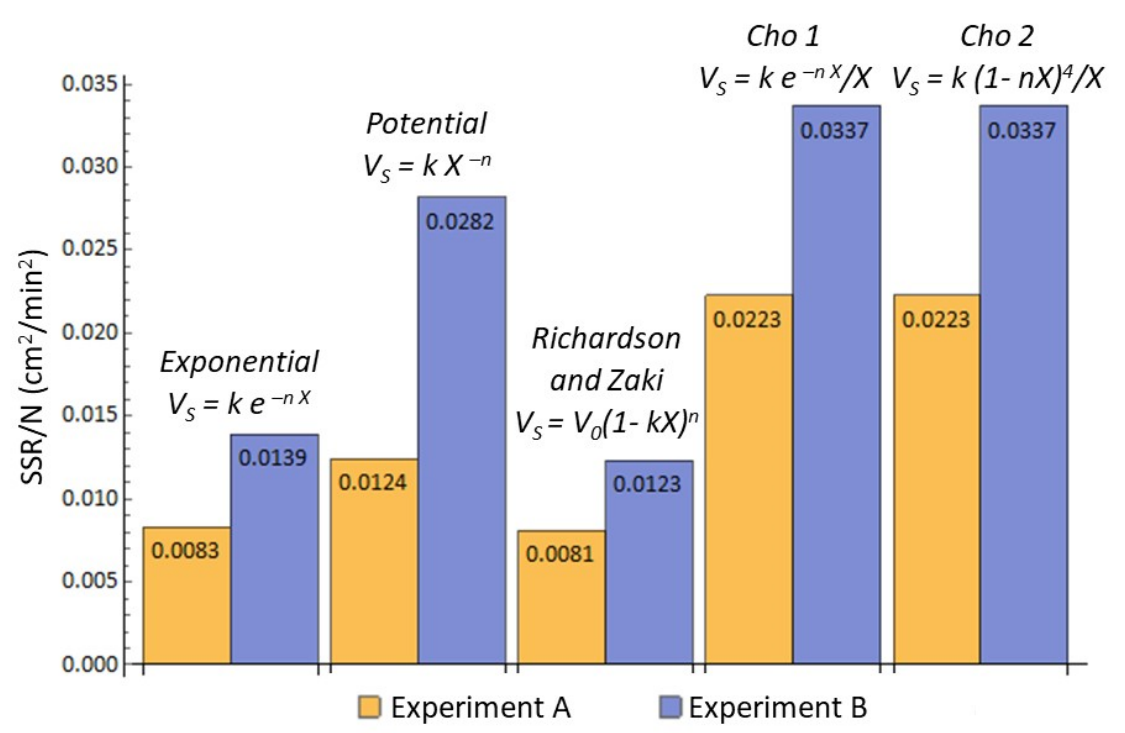

(b)

Fig. S3. Mean Sum of Squares Residuals (SSR/N) of the settling velocity adjustments for experiments A and B. (a) Initial settling velocity (b) Final settling velocity.

\section{REFERENCES}

[1] G.A. Ekama, J.L. Barnard, F.W. Günthert, P. Krebs, D.S. McCorquodale, J. A. Parker, E.J. Wahlberg, Secondary settling tank: theory, modelling, design and operation.

Scientific and Technical Report No. 6. IAWQ, International Association on Water 
Quality, London, 1997.

[2] A.O. Al-Jasser, Enhancement of Sludge Settling with Chemical Additives, Water Environ. Res. 81 (2009) 849-857. doi:10.2175/106143008X390799.

[3] A. Vanderhasselt, A. Fuchs, P. Vanrolleghem, G. Staudinger, W. Verstraete, Monitoring of the Effects of Additives on Sludge Separation Properties by Using Sensors, Water Environ. Res. 71 (1999) 355-362.

[4] R. Font, M. Pérez, Hydrodynamic parameters from the Michaels and Bolger method, Chem. Eng. J. 80 (2000) 167-175. doi:10.1016/S1383-5866(00)00088-5.

[5] G.W. Chen, I.L. Chang, W.T. Hung, D.J. Lee, Regimes for zone settling of waste activated sludges, Water Res. 30 (1996) 1844-1850. doi:10.1016/0043-1354(95)003223.

[6] Y.Q. Zhao, Settling behaviour of polymer flocculated water-treatment sludge I: analyses of settling curves, Sep. Purif. Technol. 35 (2004) 71-80. doi:10.1016/S13835866(03)00132-1.

[7] Y.Q. Zhao, Settling behaviour of polymer flocculated water-treatment sludge II: Effects of floc structure and floc packing, Sep. Purif. Technol. 35 (2004) 175-183. doi:10.1016/S1383-5866(03)00133-3.

[8] L.J. Teece, J.M. Hart, K.Y. Ni Hsu, S. Gilligan, M.A. Faers, P. Bartlett, Gels under stress: The origins of delayed collapse, Colloids Surfaces A Physicochem. Eng. Asp. 458 (2014) 126-133. doi:10.1016/j.colsurfa.2014.03.018.

[9] P. Bartlett, L.J. Teece, M.A. Faers, Sudden collapse of a colloidal gel, Phys. Rev. E. 85 (2012) 1-13. doi:10.1103/PhysRevE.85.021404.

[10] M.L. Kilfoil, E.E. Pashkovski, J.A. Masters, D.A. Weitz, Dynamics of weakly aggregated colloidal particles., Philos. Trans. R. Soc. A Math. Phys. Eng. Sci. 361 
(2003) 753-766. doi:10.1098/rsta.2002.1163.

[11] P. François, F. Locatelli, J. Laurent, K. Bekkour, Experimental study of activated sludge batch settling velocity profile, Flow Meas. Instrum. 48 (2016) 112-117. doi:10.1016/j.flowmeasinst.2015.08.009.

[12] R.B. Baird, A.D. Eaton, E.W. Rice, L. Bridgewater, American Public Health Association, A.W.W. Association, W.E. Federation, Standard methods for the examination of water and wastewater, 23rd ed., American Public Health Association, Washington DC, 2017.

[13] I. Nopens, Modelling the activated sludge flocculation process: a population balance approach, 2005. http://lib.ugent.be/fulltxt/RUG01/000/894/656/RUG01000894656_2010_0001_AC.pdf(accessed March 3, 2017).

[14] H. Jang, A.J. Schuler, The Case for Variable Density: A New Perspective on Activated Sludge Settling, Water Environ. Res. 79 (2007) 2298-2303. doi:10.2175/106143007X194347.

[15] D.P. Mesquita, O. Dias, A.L. Amaral, E.C. Ferreira, A Comparison between bright field and phase-contrast image analysis techniques in activated sludge morphological characterization., Microsc. Microanal. 16 (2010) 166-174. doi:10.1017/S1431927609991358.

[16] J. De Clercq, F. Jacobs, D.J. Kinnear, I. Nopens, R.A. Dierckx, J. Defrancq, P.A. Vanrolleghem, Detailed spatio-temporal solids concentration profiling during batch settling of activated sludge using a radiotracer, Water Res. 39 (2005) 2125-2135. doi:10.1016/j.watres.2005.03.023.

[17] B. Li, M.K. Stenstrom, Research advances and challenges in one-dimensional modeling of secondary settling Tanks - A critical review, Water Res. 65 (2014) 40-63. doi:10.1016/j.watres.2014.07.007. 
[18] J.F. Richardson, W.N. Zaki, Sedimentation and fluidisation: Part I, Trans. Inst. Chem. Eng. 32 (1954) 35-53. doi:10.1016/S0263-8762(97)80006-8.

[19] R. Font, P. García, M. Rodriguez, Sedimentation test of metal hydroxides: Hydrodynamics and influence of pH, Colloids Surfaces A Physicochem. Eng. Asp. 157 (1999) 73-84. doi:10.1016/S0927-7757(99)00091-6.

[20] J. Garside, M.R. Al-Dibouni, Velocity-Voidage Relationships for Fluidization and Sedimentation in Solid-Liquid Systems, Ind. Eng. Chem. Process Des. Dev. 16 (1977) 206-214. doi:10.1021/i260062a008.

[21] M. Johnson, J. Peakall, M. Fairweather, S. Biggs, D. Harbottle, T.N. Hunter, Characterization of Multiple Hindered Settling Regimes in Aggregated Mineral Suspensions, Ind. Eng. Chem. Res. 55 (2016) 9983-9993. doi:10.1021/acs.iecr.6b02383.

[22] H.Y. Chung, D.J. Lee, Porosity and interior structure of flocculated activated sludge floc, J. Colloid Interface Sci. 267 (2003) 136-143. doi:10.1016/S0021-9797(03)00682-9.

[23] C.P. Chu, D.J. Lee, X.F. Peng, Structure of conditioned sludge flocs, Water Res. 38 (2004) 2125-2134. doi:10.1016/j.watres.2004.02.003.

[24] F. Xiao, K.M. Lam, X.Y. Li, Investigation and visualization of internal flow through particle aggregates and microbial flocs using particle image velocimetry, J. Colloid Interface Sci. 397 (2013) 163-168. doi:10.1016/j.jcis.2013.01.053.

[25] M. Hriberšek, B. Žajdela, A. Hribernik, M. Zadravec, Experimental and numerical investigations of sedimentation of porous wastewater sludge flocs, Water Res. 45 (2011) 1729-1735. doi:10.1016/j.watres.2010.11.019.

[26] M.C. van Loosdrecht, P.H. Nielsen, D. Lopez-Vazquez, C. M. Brdjanovic, Experimental Methods in Wastewater Treatment, IWA Publishing, London, 2016.

[27] B. Buratto, S.P. Usher, D. Parris, P.J. Scales, Wall effects during settling in cylinders, 
Colloids Surfaces A Physicochem. Eng. Asp. 449 (2014) 157-169.

doi:10.1016/j.colsurfa.2014.02.045.

[28] R. Spehar, A. Kiviti-Manor, P. Fawell, S.P. Usher, M. Rudman, P.J. Scales, Aggregate densification in the thickening of flocculated suspensions in an un-networked bed, Chem. Eng. Sci. 122 (2015) 585-595. doi:10.1016/j.ces.2014.10.018. 